\title{
Polysaccharide Preparation from Bacterial Cells
}

National Cancer Institute

\section{Source}

National Cancer Institute. Polysaccharide Preparation from Bacterial Cells. NCI

Thesaurus. Code C113047.

Separation and purification of bacterial membrane polysaccharides from target bacterial cultures. 